\title{
MOOC “Competências digitais para professores": uma prática formativa inovadora
}

\section{MOOC "Digital Competences for teachers": an innovative training practice}

\author{
António Moreira Teixeira \\ Branca Alberto de Miranda \\ Isolina Pereira de Oliveira \\ Maria do Carmo Teixeira Pinto \\ Universidade Aberta UAb e Laboratório de Educação \\ e Ensino a Distância LE@D, (Portugal)
}

\section{Resumo}

Os Massive Open Online Courses (MOOC) são cursos abertos disponibilizados online, sem custos para o utilizador e desenhados de forma a poderem ser escalados, permitindo um grande número de participantes. Neste âmbito, uma equipa de investigadores da UAb desenvolveu um modelo pedagógico que tem vindo a ser testado, debatido e aprofundado, o qual assenta em pressupostos socioconstrutivistas que combinam a dimensão da autoaprendizagem com a dimensão social. Neste artigo analisa-se e discute-se em que medida a aplicação e aprofundamento deste modelo pedagógico no MOOC Competências Digitais para Professores promove a apropriação de conhecimento e partilha de experiências, bem como o desenvolvimento de competências digitais. Considerando que a aprendizagem se desenvolve através da interação com os materiais, os facilitadores e entre os participantes, procedeu-se à análise das interações ocorridas no espaço virtual; numa segunda fase, realizou-se uma análise de conteúdo das diferentes produções dos participantes. Em conclusão, o artigo evidencia a contribuição deste modelo pedagógico inovador para a apropriação de novos conhecimentos e a reflexão sobre as práticas, destacando a possibilidade destes cursos se constituírem como um instrumento poderoso na formação de professores e outros profissionais, proporcionando pontos de reflexão sobre as novas abordagens pedagógicas que emergem a partir dos MOOCs.

Palavras-chave: competências digitais; elearning; interação virtual; modelo pedagógico; MOOC.

\section{Abstract}

Massive Open Online Courses (MOOC) are open courses available online without costs for the user and designed in such a way as to be scalable, thus allowing for a large number of participants. In this framework, a team of researchers from Universidade Aberta (UAb.pt) has 
developed a specific pedagogical model which has being tested, discussed and improved. This model is based on a number of socio-constructivist principles which combine autonomous learning with a strong social dimension. This paper analyses and discussed to which extent the use and improvement of the model in the MOOC Digital Competencies for Teachers promotes knowledge appropriation and the sharing of experiences, as well as the development of digital competencies. Taking into consideration that learning is conducted through interaction with materials, facilitators and with fellow participants, the research starts by analyzing interactions which took place in the virtual learning environment. In a second phase, it is conducted a content analysis of the different digital artefacts produced by the participants. In conclusion, the paper shows how this innovative model contributes to the appropriation of new knowledge and reflection on practices, highlighting the possibility of these courses being powerful tools for the training of teachers and other professionals. The authors also discuss the new pedagogical approaches emerging from MOOCs.

Keywords: Digital competencies; eLearning; online interaction; pedagogical model; MOOC.

Os Massive Open Online Courses (MOOCs) são cursos de eLearning, de acesso aberto, a que acresce a característica de serem escaláveis, com vista a servir um número elevado e indeterminado de participantes. Pelo facto de serem abertos, estes cursos são destinados a todos, não sendo exigidas qualificações de entrada, e proporcionando a experiência de um curso completo, totalmente online e gratuito (EADTU, 2015).

Pela conjugação da abertura e da escalabilidade, os MOOCs oferecem um acesso à aprendizagem sem precedentes. Neste sentido, tem sido defendido que contribuem para a equidade social, ao permitir o acesso de muitas populações a uma educação superior de qualidade, ainda que o seu impacto socioético esteja ainda por investigar (Rolfe, 2015). De igual modo, ao nível das práticas pedagógicas, estes cursos também colocam desafios interessantes pelo que proporcionam em termos da autonomia dos participantes na construção dos seus próprios percursos de aprendizagem desenvolvidos em espaços de aprendizagem em rede.

Os MOOCs constituem um fenómeno recente, iniciado em 2008, com George Siemens, Stephen Downes e Dave Cormier (Downes, 2012; Daniel, 2012; Watters, 2012). Inicialmente desenhados de acordo com os princípios da teoria conectivista (Downes, 2012; Siemens, 2012, Cormier, 2010), e no seguimento das experiências de Alec Couros ${ }^{1}$ e David Wiley ${ }^{2}$, foi com as iniciativas posteriores conduzidas por Sebastian Thrun, Peter Norvig, Daphne Koller e Andrew Ng, em particular com a decisão de Thrun e Norvig de abrirem o acesso ao seu curso "An Introduction to AI", em 2011, que o fenómeno se tornou global (Daniel, 2012). Esta dicotomia entre os MOOCs de inspiração conectivista (cMOOC) e os baseados numa perspetiva mais tradicional (xMOOC) marcou o desenvolvimento do fenómeno.

A primeira experiência em língua portuguesa, levada a cabo por João Mattar e Paulo Simões, no final de 2012, organizou-se de acordo com uma perspectiva 
conectivista e não institucional. Seguiu-se a iniciativa universitária liderada pela Universidade Aberta (UAb), em 2013, no âmbito da primeira aliança transnacional formada para a oferta de MOOCs, a OpenUp Ed, coordenada pela EADTU e com o patrocínio da Comissão Europeia. Foi no âmbito deste projeto pioneiro que a UAb concebeu o modelo iMOOC, o primeiro modelo pedagógico institucional desenhado especificamente para MOOCs, que procurou encontrar um compromisso entre as duas tendências dominantes na altura (cMOOC e xMOOC).

$\mathrm{O}$ modelo iMOOC funda-se numa perspetiva integrada da aprendizagem que agrega a promoção da responsabilidade individual e das relações interpessoais, num ambiente inovador que facilite a interação e a inclusão (Teixeira \& Mota, 2013). Procurou-se dar resposta aos desenvolvimentos emergentes no âmbito das práticas educacionais abertas pela construção de cenários de desenvolvimento de aprendizagens pessoais em comunidades online e redes sociais. A implantação deste modelo exigiu o desenho de um ambiente inovador que nasceu da integração de duas plataformas virtuais pré-existentes: Elgg e Moodle. O objetivo foi desenvolver um espaço de aprendizagem com base na interação social, assegurado pela Elgg, a que se agrega, no espaço Moodle, toda a informação sobre o curso (conteúdos, recursos de aprendizagem, orientações de trabalho, calendarização), bem como fóruns de dúvidas e uma ferramenta para avaliação por pares. Fiel aos princípios da aprendizagem em rede, a base do trabalho realiza-se na interação ocorrida na Elgg, onde os participantes constroem os seus personal learning environments (PLEs) institucionais (Teixeira \& Mota, 2013).

$\mathrm{Na}$ criação do iMOOC estiveram presentes pressupostos como a combinação da dimensão da autoaprendizagem com a dimensão social, visando tornar as experiências de aprendizagem mais ricas e gratificantes. Houve, também, a preocupação de articular a flexibilidade proporcionada pela educação a distância online com o apoio necessário à concretização das tarefas. Assume-se o propósito de alargar a aprendizagem ao maior número possível de pessoas e, sobretudo, trazêlas para o ambiente digital pela presença que o mesmo tem atualmente na vida quotidiana (Teixeira \& Mota 2013, 2014a).

A aprendizagem é centrada no participante e baseada na realização de atividades, evidenciando-se através da criação de produtos de acesso aberto (textos, vídeos, apresentações, etc.), que demonstrem os conhecimentos e competências dos participantes relativamente aos temas em estudo. Nesste modelo, espera-se que os participantes não só assumam um papel ativo na sua própria aprendizagem, mas também sejam suporte da comunidade de aprendizagem, mediante o diálogo, a colaboração e o feedback interpares. A avaliação formativa revela-se mediante contribuições nos blogs, construção de artefactos e do feedback entre pares.

A presença do professor expressa-se pela disponibilização do Guia de Aprendizagem e das orientações detalhadas para as atividades apresentadas, assim como pela proposta de exploração de recursos e pelo feedback semanal. O modelo iMOOC contempla a existência de uma equipa de facilitadores, que complementam 
a ação dos professores autores dos cursos, com vista a apoiar os participantes, à qual cabe reunir informação relevante no sentido de monitorizar o processo de aprendizagem nas redes virtuais.

O iMOOC constituiu a base de um modelo posterior, o sMOOC, desenvolvido, em 2014, no âmbito do projeto europeu Elearning, Communication and Open-data: Massive Mobile, Ubiquitous an Open Learning (ECO). Este modelo de MOOC, social e seamless, procura melhorar a qualidade da experiência de aprendizagem pelo reforço da componente social de apoio aos participantes, tal como pela promoção do acesso a partir de múltiplas plataformas de aprendizagem e dispositivos móveis, assegurando uma aprendizagem ubíqua, abrangente e contextualizada. De igual modo, o modelo aspira ainda a promover a relação da aprendizagem com a experiência de vida dos participantes mediante a contextualização dos conteúdos, suportando estratégias de aprendizagem adaptativas, através da utilização de aplicativos móveis e de gamificação. (Brouns et al., 2014; Osuna \& Camarero, 2016).

A combinação destes modelos (sMOOC e iMOOC) serviu de referência ao desenho do MOOC Competências Digitais para Professores, o qual é analisado neste artigo. $\mathrm{Na}$ investigação apresentada, analisa-se como é que estas abordagens pedagógicas emergentes contribuem para novas formas de aprendizagem e de regulação das aprendizagens. O estudo foca-se no envolvimento dos participantes nas atividades propostas e os diferentes tipos de interações ocorridas na rede. Além disso, apontamse pistas para a aplicabilidade dos princípios e conceitos conectivistas (Downes, 2012; Siemens, 2005) no desenvolvimento das referidas abordagens pedagógicas, enfatizando o papel dos participantes nos seus percursos de aprendizagem e na interação entre os participantes.

\section{METODOLOGIA}

Neste estudo, analisam-se os resultados da aplicação do modelo pedagógico dos sMOOCs, particularmente no que se refere ao modo como decorreu a interação entre os participantes do curso. Este modelo pressupõe que a aprendizagem se desenvolve através da interação com os materiais (disponibilizados nos tópicos ou produzidos pelos participantes) e com os facilitadores, considerando que as interações ocorridas no espaço virtual devem propiciar a apropriação, reelaboração e produção de novos conteúdos (Brouns et al., 2014). Parte-se, assim, do pressuposto que as trocas realizadas pelos participantes são um veículo da aprendizagem e contribuem para o seu desenvolvimento profissional (Tillema, Van Der Westhuizen \& Smith, 2015).

A metodologia do estudo assenta no modelo design-based research. Esta metodologia apresenta potencialidades quando se pretende criar e expandir conhecimento relacionado com o desenvolvimento, a implementação e a sustentação de ambientes de aprendizagem inovadores" (The design-based research collective, 2003). O desenvolvimento e a investigação ocorrem em cenários autênticos e realizase através de ciclos contínuos de design, implementação, análise, reflexão e redesign. 
O MOOC Competências Digitais para Professores foi desenhado de acordo com o modelo sMOOC e teve 5 edições. Com efeito, o processo desenvolveu-se em 4 fases que ocorreram ao longo de três anos: $1^{\text {a }}$ ) Design do Curso $(2014) ; 2^{0}$ ) Iterações do curso com 3 edições em 2014 e 2015; $3^{\text {a }}$ ) Avaliação do curso; $4^{\text {a }}$ ) Re-Design do curso e sua aplicação em novas edições em 2016 (figura 1).

Figura 1. Ciclos da investigação

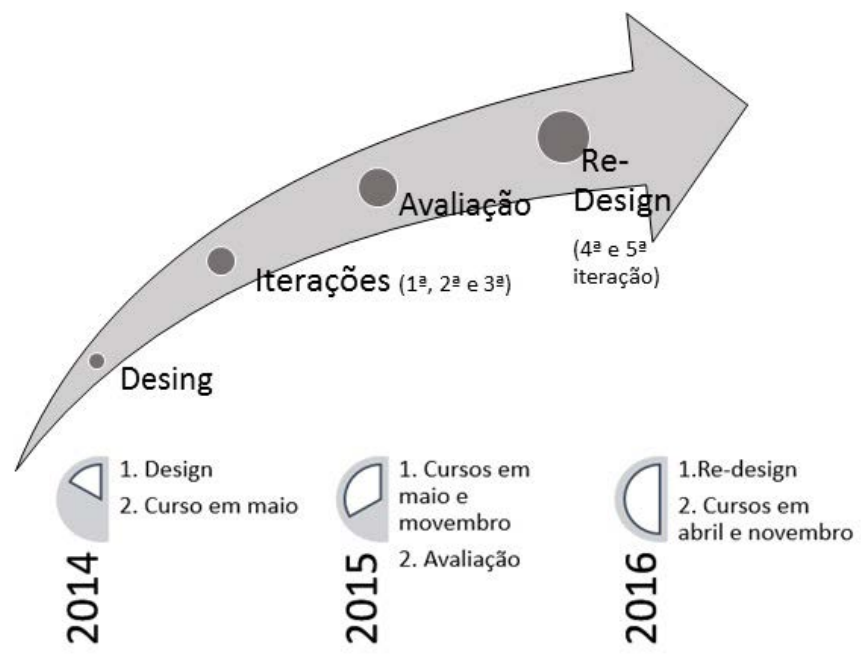

Assim, em um primeiro momento, foi desenhado o MOOC Competências Digitais para Professores com base numa combinação dos modelos SMOOC e iMOOC. Num segundo momento, foram redesenhadas e/ou ajustadas atividades, visando a evolução dessas abordagens e reunidos dados que permitiram fazer uma análise sistemática dos processos e dos modos pelos quais se desenvolvem. Pretendendo ampliar a interação no seio da comunidade, os professores responsáveis pelo design do curso introduziram na edição seguinte alterações no modo de produção dos feedbacks, procurando-se dar uma resposta mais eficaz às necessidades da comunidade de aprendizagem. Por outro lado, com a inclusão de algumas ferramentas adicionais, como a barra de progresso e os crachás (badges), procurou-se também elevar os níveis de motivação dos participantes, bem como aumentar os níveis de interação.

Por fim, no terceiro momento, que corresponde à análise retrospetiva ainda em curso, procedeu-se à análise completa dos dados recolhidos.

A $3^{\text {a }}$ edição do MOOC Competências Digitais para Professores, que constituiu o objeto de estudo neste artigo, decorreu em novembro de 2015, contando com cerca de 400 participantes. A quase totalidade destes participantes era falante de português e a esmagadora maioria professores, que tinham como objetivos 
desenvolver competências na área das TICs e, principalmente, saber utilizá-las em contextos educativos. A equipa responsável incluiu 4 professores, que produziram os conteúdos e supervisionaram a aprendizagem, e 2 facilitadoras.

O curso teve a duração de 5 semanas e encontrava-se organizado em 4 tópicos, sendo o primeiro um módulo de ambientação (Boot Camp) e os seguintes centrados em três temas distintos. Durante o módulo de ambientação desenvolvem-se atividades de familiarização com o ambiente de aprendizagem e com as formas de comunicação, interação e publicação no curso. Os participantes foram desafiados a completar determinadas tarefas, tendo em vista o desenvolvimento de competências que lhes permitam otimizar a utilização das ferramentas disponíveis nos espaços onde ocorreram as interações (Moodle e Elgg) e, consequentemente, construir o seu próprio PLE.

Em cada tema foram disponibilizados diversos tipos de Recursos Educacionais Abertos (REAs) e foi sugerido aos participantes que pesquisassem, partilhassem e debatessem, incentivando-os a utilizarem diversas ferramentas como blogs, Curtas, Favoritos e Fóruns. Em dois momentos do curso é proposto aos participantes a construção de artefactos digitais, produtos que foram avaliados pelos pares.

Este estudo, de natureza qualitativa (Bogdan \& Biklen, 2013; Amado, 2014), utiliza como dados as interações ocorridas nos diversos fóruns bem como nos PLEs dos participantes, recorrendo-se à técnica da análise de conteúdo (Bardin, 2012; Amado 2014). A interação é baseada na linguagem e a sua análise permitiu descobrir como os participantes desenvolveram a sua compreensão e realizaram a aprendizagem em cenários online (Suthers, Dwter, Medina \& Vatrapu, 2009). Neste sentido, foram analisadas: i) interações ocorridas entre os participantes nos três temas do curso (Elgg); ii) posts publicados nos fóruns disponíveis nos diversos temas, bem como os comentários dos participantes sobre a avaliação por pares dos artefacto produzidos (Moodle); iii) interações decorrentes do feedback dado ao longo do curso, pelos facilitadores, pelos professores e pelos participantes às atividades que foram desenvolvidas (Moodle).

\section{INTERAÇÃO SOCIAL NO AMBIENTE VIRTUAL ELGG}

A componente Elgg do ambiente de aprendizagem disponibiliza um conjunto diversificado de ferramentas que permitem publicar, debater, selecionar temas e seguir outros participantes. Foram analisadas todas as mensagens colocadas pelos participantes (cujas falas são identificadas pelas iniciais dos seus nomes), no decorrer dos temas 1, 2 e 3, nas Curtas (413), nos Blogs (140) e nos Favoritos (99).

O Tema 1 - Pesquisar, selecionar e partilhar recursos na Internet, pretendeu proporcionar uma aproximação à temática do MOOC Competências Digitais para Professores, centrando-se a reflexão e debate em torno de algumas questões suscitadas pela utilização dos recursos disponíveis na Internet, designadamente 
a qualidade das fontes de informação e os problemas éticos suscitados pela sua utilização.

No decurso da $1^{\text {a }}$ fase de trabalho, os participantes foram convidados a explorar, de forma individual, os diferentes recursos disponibilizados. Seguidamente, cada participante foi solicitado a partilhar com os seus pares as questões, interrogações e comentários que a análise e reflexão sobre recursos lhe tivessem suscitado.

Numa $2^{\mathrm{a}}$ fase, foi proposto a cada participante que produzisse um comentário sobre a atual relevância dos aspetos abordados no tema, bem como selecionasse contribuições de outros participantes e as comentasse.

Relativamente ao interesse suscitado pelos recursos disponibilizados verificouse que a comunidade dispensou bastante atenção ao vídeo que apresenta de forma sintética, mas muito clara, a ideia-chave expressa por Carr (2011): a Internet está a tornar-nos pensadores mais superficiais. A maioria dos participantes que comentaram o vídeo expressou concordância com estas posições. Houve quem se questionasse se o facto de a informação ser "mais veloz que o vento" e chegar até nós mediante um simples clique "Será assim tão bom..." (Curtas IC) e quem considerasse que "A Internet pode viciar ao ponto de se deixar de ter uma vida saudável" (Curtas A). Contudo, a "solução" para evitar os eventuais aspectos negativos decorrentes da utilização da Internet poderá estar em "Saber utilizar, como em tudo, com conta peso e medida...” (Curtas PR).

No entanto, a abundância de recursos disponíveis na Internet e a facilidade de acesso aos mesmos, pode propiciar a apropriação e reprodução indevida dos mesmos, ou seja, o plágio. Esta questão, bem como a do papel da Internet na nossa vida, em geral, e na educação, em particular, suscitou ampla discussão. Relativamente ao plágio os participantes questionaram-se sobre as razões justificativas deste tipo de comportamento - pressões de ordem vária variada e/ou o efetivo desconhecimento do que significa e comporta o ato de plagiar - e discutiram estratégias capazes de reduzir a prática do plágio. No âmbito da discussão foram colocadas muitas mensagens Curtas, nas quais se abordou a relação plágio/ética, questão que é objeto de uma reflexão mais alargada em "O Plágio: considerações, proposta e experiências práticas" (Blog GJ).

No decurso da semana dedicada a este tema, registou-se uma importante partilha de ferramentas web 2.0 para criação de conteúdos, detectores de plágio e repositórios de recursos para professores, para além de links para artigos e e-books relacionados com o tema em estudo.

O Tema 2 - Utilizar competências digitais na sala de aula pretendeu provocar o debate sobre as potencialidades educativas das ferramentas digitais e proporcionar um espaço de análise e de partilha de práticas pedagógicas, que envolvessem estas ferramentas, tendo em vista a elaboração de propostas de atividades de sala de aula com um potencial inovador. A exploração e partilha de recursos sobre a temática centrou-se nas intencionalidades subjacentes à utilização das ferramentas digitais: criar ambientes de aprendizagem mais apelativos e motivadores e propiciar o 
desenvolvimento de diversas competências, tendo em vista a eficácia do processo educativo. A partilha de recursos como software, sites e documentos, deu origem a um debate sobre o papel das tecnologias digitais na educação destacando-se que estas "facilitam a pesquisa, a comunicação e a divulgação em rede" (Curta MR), "apresentam muitas oportunidades para os estudantes produzirem informação e conhecimento" (Curta de JF), podendo ter um importante papel na aprendizagem "na medida em que os ajuda [os estudantes] a construir o conhecimento" (Blog MA).

Sobre este tema, foi também destacada a necessidade de conhecer as ferramentas disponíveis e de saber como potencializar o seu uso na sala de aula: "é preciso conhecer as especificidades dos artefactos para então poder explorar ao máximo o seu potencial educativo" (Curta PP). Também foram referidas algumas barreiras à utilização das tecnologias nas escolas como "a escassez de software e recursos digitais de qualidade (...) [e a] resistência (...) à inovação" (Blog IC). No entanto, as tecnologias digitais podem ser um fator capaz de despoletar a inovação educativa, uma vez que "os ambientes virtuais [podem] incentivar a colaboração e partilha, a mudança das pedagogias...” (Blog GJ), mas esta mudança implica uma nova postura por parte do professor: "parece que o futuro do ensino passa por formar um novo tipo de professor para um novo tipo de estudante!” (Blog GJ). Foi sublinhado que só a formação permitirá quebrar a resistência à mudança «a sociedade digital precisa de novos perfis profissionais». (Blog IS).

A discussão sobre a intencionalidade do uso de ferramentas digitais constituiuse como o foco central do debate do tema 2: "o tema 2 deu-me a oportunidade (...) de refletir sobre a prática pedagógica envolvendo o recurso a ferramentas tecnológicas, nomeadamente, na seleção daquelas que de forma mais adequada poderão contribuir para facultar aprendizagens significativas aos alunos" (Blog MR).

O Tema 3 - Promover a aprendizagem colaborativa com ferramentas digitais partiu do pressuposto que a maior parte dos participantes já tinha estado envolvida em situações de aprendizagem colaborativa mediadas por ferramentas digitais. Com efeito, as atividades propostas pretenderam provocar o debate em torno dos desafios da disseminação do uso das tecnologias digitais no quotidiano das pessoas e da sua relação com os novos modos de aprender. Deste modo, ao solicitar-se a descrição de situações vividas, contribuiu-se para o confronto de experiências e pontos de vista e promoveu-se a interação entre os participantes. O uso e a opção por uma dada ferramenta digital na abordagem de um tema suscita um conjunto de questões que é importante partilhar e debater. A inclusão dessas ferramentas nas práticas educativas pressupõe o conhecimento das suas potencialidades e constrangimentos. Daí ter sido relevante analisar os contributos trazidos para a aprendizagem. A discussão e a partilha de experiências, receios e sucessos contribuiu para o desenvolvimento de um olhar crítico sobre o modo como são utilizadas essas ferramentas e como se pode potenciar o seu uso.

O debate centrou-se em torno dos conceitos de cooperação e colaboração, partindo do texto "Colaboração/cooperação: conceitos e preconceitos" com a partilha 
de recursos nas curtas e nos blogs sobre a divulgação de links, como por exemplo a "criação de ambientes de aprendizagem colaborativa" (Curta AF), o "facebook e a aprendizagem colaborativa" (Curta NF), "modelo de ensino colaborativo/ cooperativo" (Blog HV) e "diferenças e semelhanças entre as aprendizagens colaborativa e cooperativa (Blog ES). No fórum, assistiu-se à divulgação de links e esquemas relacionados com a aprendizagem colaborativa, embora tenha prevalecido a apresentação de definições propostas por diversos autores, como Dillenbourg (1999), e a importância do trabalho colaborativo entre os professores.

Numa fase seguinte, os participantes dialogaram sobre a wiki, pouco utilizada como recurso educativo, como se depreende das intervenções. O texto disponibilizado "Práticas de avaliação formativa em ambiente wiki" apresentou o trabalho desenvolvido por um professor com alunos com 10-11 anos de idade, centrado na elaboração de um guião de entrevista num ambiente wiki. Nele faz-se uma breve apresentação da ferramenta, das suas potencialidades e limitações, o que provocou nos participantes a pesquisa e partilha nas curtas e nos blogs de afirmações que pretendiam clarificar o significado da referida ferramenta, bem como o relato de experiências onde tinha sido usada. "O termo wiki é utilizado para definir o software colaborativo que cria coleções de páginas interligadas formando um hipertexto ou uma hipermídia". (Curta IC). "Uma das vantagens da wiki em educação é favorecer a revisão do trabalho à medida que este se elabora" (Curta EA).

O texto "As redes sociais no apoio ao ensino presencial" despoletou sobretudo a pesquisa em torno de estudos disponíveis na internet sobre como aprender através das redes sociais e alargou o debate nos blogs aos desafios colocados aos professores pela chamada geração digital. Por exemplo, a participante IC publicou na curta um link para o artigo "As redes sociais na promoção da aprendizagem colaborativa: um estudo no ensino secundário”.

De entre os Favoritos que foram partilhados referentes ao tema 3, a maior parte dos artigos abordaram a aprendizagem colaborativa, o papel do professor e, também, alguns recursos e ferramentas potencializadores de aprendizagem colaborativa.

\section{INTERAÇÕES NA PLATAFORMA VIRTUAL MOODLE}

Sob este título apresenta-se a análise do feedback prestado pelos membros participantes da comunidade (professores, facilitadores e participantes) às atividades desenvolvidas, com base nas mensagens colocadas no espaço virtual Moodle.

Os fóruns virtuais tiveram como objetivo esclarecer eventuais dúvidas, relacionadas com a utilização das ferramentas disponibilizadas e com a gestão do curso, assim como proporcionar a interação entre os participantes, e foram moderados pelos facilitadores do curso.

Para se compreender o papel do feedback analisaram-se os posts nos fóruns disponíveis durante a ambientação (fórum 1) e nos diversos temas (fóruns 2, 3 e 
4). Foram publicados 322 posts nos 4 fóruns disponibilizados e dinamizados pelos facilitadores.

Relativamente ao fórum 1, foram abordadas questões relacionadas com a gestão da interação, com a familiarização com as ferramentas a utilizar ao longo do curso e com a avaliação e certificação do curso, como se apresenta de seguida:

- gestão das plataformas virtuais - como se gere o trabalho nos dois ambientes virtuais (Moodle e Elgg);

- gestão da interação - como seguir outros participantes ou os favoritos;

- utilização de ferramentas - o acesso aos blogs, a inserção de imagens nas mensagens, a receção de notificações no email, o uso da barra de editor de texto;

- procedimentos sobre a avaliação - sobretudo, aspetos ligados à creditação e certificação do curso.

O tempo de resposta às questões colocadas pelos participantes foi sempre inferior a 24 horas. A prontidão é crucial para um prosseguimento eficaz das atividades propostas. Destaca-se que a iniciativa de responder a algumas questões partiu, frequentemente, de outros participantes que já conseguiam dominar os procedimentos.

A análise das mensagens dos fóruns disponibilizadas nos temas 1, 2 e 3 permitiu identificar 5 categorias, como se pode observar no Quadro 1.

Quadro 1. Categorização das questões colocadas nos fóruns 2, 3 e 4

\begin{tabular}{|l|l|l|}
\hline \multicolumn{1}{|c|}{ Categorias } & \multicolumn{1}{|c|}{ Exemplos } & \multicolumn{1}{c|}{$\begin{array}{c}\text { \% de } \\
\text { posts }\end{array}$} \\
\hline Informações gerais & $\begin{array}{l}\text { Prazos de atividades } \\
\text { Certificação } \\
\text { Acesso aos questionários }\end{array}$ & 22,3 \\
\hline Recursos tecnológicos & $\begin{array}{l}\text { Acesso a recursos } \\
\text { Elaboração de artefactos } \\
\text { Utilização do blog }\end{array}$ & 20,9 \\
\hline Avaliação & $\begin{array}{l}\text { Avaliação por pares } \\
\text { Apreciação sobre o curso }\end{array}$ & 15,5 \\
\hline Conteúdos de aprendizagem & Discussão sobre as temáticas do curso & 36,5 \\
\hline Socialização & $\begin{array}{l}\text { Mensagens gerais (festividades, } \\
\text { despedidas) }\end{array}$ & 4,9 \\
\hline
\end{tabular}

O Quadro 1 evidencia que as categorias que se relacionam com o funcionamento e o desenvolvimento das atividades propostas no curso, quando consideradas no seu conjunto, foram preponderantes (as três primeiras categorias representam $58,7 \%$ das mensagens). Contudo, e apesar de ser pressuposto que a discussão 
entre os participantes ocorresse no ambiente Elgg, através da publicação nos PLEs, um conjunto de mensagens ainda significativo $(36,5 \%)$ versou sobre as temáticas do curso. Neste caso, as facilitadoras sugeriram a sua republicação no ambiente apropriado.

Foi proporcionado feedback descritivo, entre pares, aos artefactos produzidos pelos participantes no tema $2\left(27^{3}\right)$ e 3 (23) tendo-se utilizado, para este efeito, uma ferramenta da Moodle que distribui aleatoriamente os artefactos pelos avaliadores. A avaliação por pares foi conduzida tendo por base uma rubrica de classificação, inspirada em Yuan \& Recker (2015), criada pelos professores do curso. Com o objetivo de tornar este processo mais eficaz e transparente, cada um dos artefactos foi avaliado por três participantes que produziram comentários avaliativos e atribuíram uma classificação ao trabalho produzido. A classificação final de cada artefacto foi calculada a partir da média obtida das classificações atribuídas.

No Quadro 2 apresentam-se exemplos de comentários avaliativos, os quais têm em conta a referida rubrica de classificação.

Quadro 2. Ilustração de comentários avaliativos sobre os artefactos

\begin{tabular}{|c|c|}
\hline $\begin{array}{l}\text { Parâmetros de } \\
\text { avaliação dos } \\
\text { artefactos }\end{array}$ & Ilustração de comentários dos pares \\
\hline Estrutura do trabalho & $\begin{array}{l}\text { “informação clara e sem problemas maiores em termos da } \\
\text { compreensão do que é veiculado"; } \\
\text { "lamento ter de dizer, mas parece-me que este trabalho } \\
\text { merecia uma boa introdução e uma boa conclusão". }\end{array}$ \\
\hline $\begin{array}{l}\text { Reflexão sobre os } \\
\text { conteúdos }\end{array}$ & $\begin{array}{l}\text { "apresenta alguns elementos de reflexão pessoal ou de } \\
\text { perspetiva crítica face ao material estudado"; } \\
\text { "não foca aspetos relevantes relativamente à questão } \\
\text { orientadora da reflexão". }\end{array}$ \\
\hline Fundamentação teórica & $\begin{array}{l}\text { "apresenta um bom conjunto de referências bibliográficas"; } \\
\text { "falta a fundamentação de seu trabalho em algum autor (s)". }\end{array}$ \\
\hline Clareza do trabalho & $\begin{array}{l}\text { "não há uma indicação clara das ferramentas digitais } \\
\text { utilizadas"; } \\
\text { "penso apenas, que poderia estar mais completo ao nível da } \\
\text { operacionalização do uso das ferramentas tecnológicas". }\end{array}$ \\
\hline
\end{tabular}

O feedback fornecido pelos participantes em relação aos artefactos produzidos pelos seus pares evidenciou a apropriação dos critérios de avaliação que lhes foram facultados. No entanto, sendo cada trabalho avaliado por três participantes, por vezes, as classificações atribuídas apresentaram discrepâncias.

Quando se compara a média das avaliações do primeiro artefacto com as do segundo verifica-se que houve uma evolução positiva, o que pode indiciar que os comentários relativos ao primeiro podem ter tido um efeito regulador, conduzindo 
a uma melhoria dos segundos artefactos. Esta inferência é apoiada por afirmações, como: "agradeço aos avaliadores do meu artefacto e espero evoluir no artefacto 2", "com a avaliação dos artefactos pude refletir e verificar os meus erros cometidos" ou "considerei muito pertinente e interessante a avaliação dos artefactos. Faz-nos refletir e buscar a melhoria do nosso desempenho".

Os comentários pessoais proferidos pelos pares avaliadores, bem como os publicados pelos avaliados relativamente à avaliação dos seus artefactos, revelaram a importância atribuída ao modo como foi praticada a avaliação por pares. Com efeito, os participantes que requereram a avaliação e respectiva certificação destacaram essa forma de avaliação como interessante, pertinente e como um processo que contribuiu para o aperfeiçoamento dos seus desempenhos.

No final de cada um dos temas os professores elaboraram um feedback a partir dos elementos recolhidos pelos facilitadores. Essa recolha baseia-se no seguinte conjunto de questões: 1) Como é que os participantes exploram os recursos disponibilizados? 2) Que conclusões se podem retirar da discussão sobre cada um dos temas e que aspectos foram privilegiados nessa discussão? 3) Que recursos pesquisaram e partilharam os participantes em cada um dos temas? 4) Como utilizaram as várias funcionalidades típicas da rede social Elgg? 5) Registaram-se, ou não, quebras no nível de interação ao longo de cada tema e, em caso afirmativo, em que momentos ocorreram?

O feedback elaborado pelos professores que, para além de considerar esses elementos, ainda teve em conta os resultados esperados na aprendizagem, o grau de participação nas atividades propostas e aspetos comunicacionais a melhorar (utilização correta de etiquetas, por exemplo).

As reações dos participantes ao feedback disponibilizado foram muito positivas: "obrigada Professora, pelas suas considerações. (...) agradeço o feedback que fez o favor de elaborar e partilhar, o qual é de uma grande riqueza» (Fórum Tema 3 MR) ou "esta síntese ajuda a conceptualizar os conceitos e estabelece uma orientação no que respeita às atividades desenvolvidas" (Fórum Tema 2 EA).

\section{CONTRIBUTOS DA INTERAÇÃO E DO FEEDBACK PARA A CONSOLIDAÇÃO DAS APRENDIZAGENS}

Discutimos, seguidamente, a abordagem pedagógica do MOOC Competências Digitais para Professores no que respeita aos tipos de interação ocorridos nos diferentes espaços virtuais e à relação desta com o grau de envolvimento dos participantes.

Como referido anteriormente, a qualidade e a intensidade da interação no seio da comunidade são centrais para avaliar o sucesso de um curso de acordo com o modelo sMOOC. Neste sentido, começamos por discutir os modos de interação entre os participantes e as ferramentas digitais usadas e, depois, a diversidade de feedbacks disponibilizados. 
O uso da plataforma Moodle, onde os participantes tiveram acesso a conteúdos, recursos, calendarização e respetivas orientações, foi acompanhado pelo acesso à Elgg, onde publicaram os seus produtos e interagiram com os seus pares. Os resultados mostram que um núcleo central da comunidade - os participantes plenos (Wenger, 1998) -, ainda que reduzido, acompanhou os três temas, criando o seu PLE, com elevado número de publicações (413 Curtas, 140 nos Blogs e 99 nos Favoritos) evidenciando, assim, o interesse pelo tema do curso.

Os participantes tinham diferentes objetivos e distintos percursos de aprendizagem, destacando-se aqueles que tiveram uma presença mais ativa na comunidade daqueles que tiveram uma presença menos visível e que procuraram o curso com outras motivações, como, por exemplo, aceder a recursos atualizados e credíveis.

A discussão em torno dos conceitos mobilizados nos diversos temas, acompanhada da partilha de recursos, demonstra o interesse dos participantes em relação ao aprofundamento dos conceitos. Assim, neste processo, assistiu-se a uma busca de recursos, visando fundamentar pontos de vista, que foram partilhados, utilizados e consultados pelos outros participantes.

Este esforço de divulgação e partilha do conhecimento foi estimulado pelas atividades propostas, assim como pelos incentivos dados pelos facilitadores. No mesmo sentido, refere-se o diálogo mantido em torno das potencialidades das ferramentas digitais, mediante a publicação de relatos de experiências sobre o seu uso. Verificou-se nesta pesquisa uma preocupação em descobrir experiências em contexto, isto é, sobre como outros utilizam essas ferramentas em ambientes formais de aprendizagem e os desafios que elas colocam. Deste modo, ocorreu "uma aprendizagem em rede" (Teixeira et al., 2015, p. 6), que resultou do interesse individual, da partilha de situações e recursos entre os participantes e do estímulo proporcionado pelo desenho do próprio curso.

Os participantes evidenciaram aprendizagem através das interações e da criação de artefactos, revelando conhecimentos, competências e um papel ativo que sustentou a comunidade de aprendizagem. Ainda que os resultados nos indiquem o envolvimento dos participantes e a ocorrência de aprendizagem, o reduzido número de artefactos submetidos pode significar que o interesse no curso teve a ver com a atualidade e presença da temática nas escolas e a possibilidade de mobilizar competências digitais necessárias à prática profissional e de partilhar recursos e experiências.

Verificou-se que a qualidade da participação, patente no elevado número de publicações e de interações na rede, na partilha de experiências e no debate de ideias, nem sempre foi acompanhada pelo interesse dos participantes na respetiva avaliação e certificação formais. Os participantes, na sua maioria professores, estavam sobretudo interessados na discussão sobre as várias questões que se colocam relativamente à utilização das tecnologias digitais, em contextos formais e não formais de aprendizagem, e de como é que estas podem ser incorporadas no 
trabalho que realizam. Com efeito, o interesse dos participantes centrou-se mais no acesso à riqueza informativa e argumentativa de um debate em larga escala sobre as temáticas em causa, com a possibilidade de partilha de práticas e experiências diversificadas, com isso consolidando pontos de vista, enriquecendo conhecimentos e realizando novas aprendizagens.

Por outro lado, ao longo do curso foram produzidos vários tipos de feedback, nos diferentes espaços virtuais, pelos professores e facilitadores do curso, bem como pelos respectivos pares, sendo este um aspeto importante na aprendizagem em ambientes de aprendizagem presencial e virtual, como referido por vários investigadores (Boud, 2010; Pereira et al., 2015).

A análise do feedback dos pares nos Blogs ou nas Curtas e dos comentários avaliativos sobre os artefactos revelou um diálogo contínuo entre os participantes, o que contribuiu para a autorregulação e autoavaliação da aprendizagem. Estes resultados permitem sustentar a teoria que o apoio à aprendizagem num MOOC assenta na comunidade de aprendizagem, através da colaboração, do diálogo, da retroalimentação pelos pares e do envolvimento ativo dos participantes no processo de aprendizagem (Teixeira \& Mota, 2013).

A avaliação no curso assumiu-se como formativa e revelou-se através de contribuições nos blogs, construção de artefactos e também de feedback entre pares relativamente aos artefactos produzidos. Na avaliação por pares, a identificação de discrepâncias nas classificações atribuídas aos artefactos, apesar da disponibilidade de uma rubrica de classificação constituiu um desafio para os participantes. Um outro aspeto relevante é que quando se comparam as avaliações do primeiro artefacto com as do segundo verifica-se que esse distanciamento diminui, o que pode significar uma melhor compreensão do processo. Este elemento tem vindo a ser discutido na equipa, dada a sua relevância para a formação de profissionais do ensino. Todavia, a certificação da completação do curso pressupõe necessariamente a elaboração de produtos que evidenciem as aprendizagens efetuadas, como os artefactos digitais, e respetiva avaliação por pares (Teixeira \& Mota, 2014b).

No que diz respeito ao feedback global construído pelos professores responsáveis pelo curso, com base numa análise criteriosa assente numa diversidade de dados, este conferiu aos participantes a possibilidade de identificarem os aspetos positivos da participação e as aprendizagens realizadas sobre o tema e, também, de modificarem formas de interação e envolvimento nas atividades, caso o desejassem.

No desenho do curso em análise procurou-se diversificar as situações de feedback, fomentando a interação e o diálogo entre os participantes, e os resultados indicam efeitos positivos em termos do seu envolvimento e do desenvolvimento de competências digitais. 


\section{CONCLUSÃO}

Neste artigo apresenta-se um estudo sobre o MOOC Competências Digitais para Professores que teve por base uma metodologia de design-based research (Cobb, et al., 2003), a qual tem o duplo objetivo de desenvolver teoria e desenhar intervenções educativas. Analisaram-se as diversas interações ocorridas em distintos espaços virtuais, com destaque para os tipos de feedback disponibilizados.

A análise dos resultados evidencia uma interação significativa no seio do espaço virtual da comunidade MOOC (interação com os recursos e interação social entre os participantes), que conduziu ao desenvolvimento de competências digitais e apropriação de conhecimentos e à produção de novos conteúdos. Esta reação positiva dos participantes às atividades propostas terá resultado de um design instrucional que procurou criar oportunidades de aprendizagem colaborativa e propor conteúdos e atividades ligados às experiências de vida dos participantes (Brouns et al., 2014; Osuna \& Camarero, 2016), bem como do feedback dado pelos professores e pelos facilitadores.

As atividades colaborativas e o feedback parecem ter conduzido a um processo reflexivo (Oliveira \& Serrazina, 2002; Ponte, 1998) que procurou intersetar o conhecimento teórico com as práticas quotidianas dos participantes. Neste sentido, pode afirmar-se que ocorreu aprendizagem através das interações assentes nas partilhas de recursos e ideias, de trocas de experiências e práticas (Tillema, Van Der Westhuizen \& Smith, 2015).

Apesar dos MOOC serem uma prática relativamente recente, os estudos realizados apontam para que esta prática inovadora tem vindo a assumir uma importância crescente no campo da educação. A partir dos resultados deste estudo podemos inferir que o modelo utilizado, assente num conjunto de princípios pedagógicos já enunciados, proporciona um espaço de análise problematizadora sobre as práticas, num ambiente de trabalho aberto e colaborativo. Deste modo, este modelo constituise como uma proposta inovadora, mais reflexiva e contextualizada, no campo da educação e da formação profissional.

Este estudo deixa questões em aberto como a necessidade de promover o alargamento do número de participantes envolvidos de modo contínuo e não apenas pontual e a melhoria dos níveis de interação entre os vários participantes. Para além disso, importa assegurar uma maior transparência para os envolvidos no processo de avaliação por pares, e finalmente, a escalabilidade dos tipos de feedback que se apresentam neste artigo, os quais se revelam claramente produtivos do ponto de vista da aprendizagem (Floratos, Guasch \& Espasa, 2015).

\section{NOTAS}

1. EC\&I 831: Social Media \& Open Education - http://eci831.wikispaces.com 
2. INST 7150 Introduction to Open Education - http://opencontent.org/wiki/index. php?title=Intro Open Ed Syllabus

3. O número reduzido de artefactos produzidos deve-se ao carácter pessoal de percurso de aprendizagem escolhido por cada participante.

\section{REFERÉNCIAS BIBLIOGRÁFICAS}

Amado, J. (Org.) (2014). Manual de Investigação Qualitativa em Educação. Recuperado de http://hdl. handle.net/10316.2/35271

Bardin, L. (2012). Análise de conteúdo. Lisboa: Edições 70.

Bogdan. R., y Biklen, S. (2003). Investigação Qualitativa em Educação: uma introdução à teoria e aos métodos. Porto: Porto Editora.

Boud, D., y Associates (2010). Assessment 2020: Seven propositions for assessment reform in higher education. Sydney: Australian Learning and Teaching Council.

Brouns, F., Mota, J., Morgado, L., Jansen, D., Fano, S., Silva, A., y Teixeira, A. (2014). A networked learning framework for effective MOOC design: the ECO project approach. In A. Teixeira y A. Szücs (Eds.), 8th EDEN Research Workshop: Challenges for research into open \& distance learning: doing things better: doing better things, 161-171. Budapest: EDEN. Recuperado de http://dspace. ou.nl/handle/1820/5544

Carr, N. (2011). The Shallows: What the Internet Is Doing to Our Brains. New York: W. Norton \& Company.

Cobb, P., Confrey, J., diSessa, A., Schauble, L., y Schauble, L. (2003). Design Experiments in Educational Research. Educational Researcher, 32(1), 9-13.
Cormier, D. (2010). What is a MOOC? [Youtube video]. December 8, 2010. Recuperado de www.youtube. com/watch?v=eW3gMGqcZQd

Creswell, J. (2013). Qualitative inquiry \& research design: choosing among five approaches. United States of America: SAGE.

Daniel, J. (2012). Making Sense of MOOCs: Musings in a Maze of Myth, Paradox and Possibility. Journal of Interactive Media In Education, 3(0). Disponível em: Recuperado de http:/ www-jime.open.ac.uk/jime/article/ view/2012-18

Dillenbourg, P. (1999). What do you mean by collaborative learning? In P. Dillenbourg (Ed) Collaborativelearning: Cognitive andComputational Approaches, 1-19. Oxford: Elsevier.

Downes, S. (2012). Creating the Connectivist Course. [Blog post]. Half an hour. Jan 6, 2012. Recuperado de http://halfanhour.blogspot. pt/2012/01/creating-connectivistcourse.htm

EADTU. (2015). Institutional MOOC strategies in Europe. EADTU. Fev. 2015. Recuperado de http:/ eadtu.eu/home/policv-areas/openeducation-and-moocs/news/248institutional-mooc-strategies-ineurope

Floratos, N., Guasch, T., y Espasa, A. (2015). Recommendations on 
Formative Assessment and Feedback Practices for stronger engagement in MOOCs. In Open Praxis, 7(2), AprilJune, 141-152. Recuperado de http:// www.openpraxis.org/index.php/ OpenPraxis/article/view/194/153

Oliveira, I., y Serrazina, L. (2002). A reflexão e o professor como investigador. In GTI (Ed.), Reflectir e investigar sobre a prática profissional, 30-42. Lisboa: APM.

Osuna, S., y Camarero, L. (2016). The ECO European Project: A New MOOC Dimension Based on an Intercreativity Environment. In TOJET: The Turkish Online Journal of Educational Technology, 15(1). 117-125. Recuperado de https://eric. ed.gov/?id=EJ1086187

Pereira, A., Oliveira, I., Tinoca, L., Pinto, M. C., y Amante, L. (2015). Desafios da avaliação digital no ensino superior. Lisboa: Universidade Aberta. LE@D, (eBookLead). Recuperado de http://hdl.handle.net/10400.2/5774

Ponte, J. (1998). Da formação ao desenvolvimento profissional. In Actas do ProfMat 98, 27-44. Lisboa: APM.

Rolfe, V. (2015). A Systematic Review of the Socio-Ethical Aspects of Massive OnlineOpen Courses. Recuperado de http://www.eurodl. $\operatorname{org} / ? \mathrm{p}=$ current\&article $=670$

Siemens, G. (2012). What is the theory that underpins our moocs? [Blog post]. elearnspace. Recuperado de http://www.elearnspace.org/ blog/2012/06/03/what-is-the-theorythat-underpins-our-moocs

Siemens, G. (2005). Connectivism: A Learning Theory for the Digital Age. International Journal of Instructional
Technology and Distance Learning, 2(1). Recuperado de http://www.itdl. org/journal/jan 05/article01.htm

Suthers, D., Dwyer, N., Medina, R., y Vatrapu, R. (2009). Exposing interactional processes in online learning. In K. Kumpulainen, C. HmeloSilver y M. César (Eds.), Investigating Classroom Interaction- Methodologies in Action (pp. 121-143). Rotterdam: Sense Publishers.

Teixeira, A., Mota, J., Morgado, L., y Spilker, M. (2015). iMOOC: Um Modelo Pedagógico Institucional para Cursos Abertos Massivos Online (MOOCs). Educação, Formação \& Tecnologias, 8 (1), 4-12. Recuperado de http://www.eft.educom.pt/index.php/ eft/article/view/465

Teixeira, A., y Mota, J. (2014a). A Proposal for the Methodological Design of Collaborative Language MOOCs. In Elena Martín-Monje, Elena Bárcena. (eds). Language MOOCs: Providing learning, transcending boundaries, 33-47. De Gruyter Open. Disponível em: http://www.degruyter.com/ viewbooktoc/product/455678

Teixeira, A., y Mota, J. (2014b). The iMOOC Pedagogical Model: Bridging the gap between non-formal and formal education. Actas del V Congreso Internacional sobre Calidad $y$ Accesibilidad de la Formación Virtual - CAFVIR 2014, 512-517. Guatemala. Recuperado de

http://www.esvial.org/cafvir2014 documentos/LibroActasCAFVIR2014. pdf

Teixeira, A., y Mota. J. (2013). Innovation and openness through MOOCs: Universidade Aberta's 
pedagogical model for non-formal online courses. In M. Paulsen y A. Szücs. On behalf of the EDEN (Eds.), Proceedings of the European Distance and E-Learning Network. Annual Conference. The Joy of Learning Enhancing Learning Experience Improving Learning Quality, 479-488. University of Oslo: Norway.

The Design-Based Research Collective. (2003). Design-based research: an emerging paradigm for educational inquiry. Educational Researcher, 32(1) 5-8.

Tillema, H., Van Der Westhuizen, G., y Smith, K. (Eds). (2015). Mentoring for learning "Climbing the Mountain". Rotterdam: Sense Publishers.
Watters, A. (2012). Top EdTech Trends of 2012: MOOCs. Hack Education. Recuperado de http:/ hackeducation.com/2012/12/03/toped-tech-trends-of-2012-moocs

Wenger, E. (1998). Communities of practice: learning, meaning, and identity. Cambridge: Cambridge University Press.

Yuan, M, y Recker, M. (2015). Not all rubrics are equal: a review of rubrics for evaluating the quality of open educational resources. IRROLD, 16(5). Athabasca University. Recuperado de http://www.irrodl.org/index.php/ irrodl/article/view/2389/3412

\section{PERFIL ACADÉMICO E PROFISSINAL DOS AUTORES}

António Teixeira. Professor na Universidade Aberta, Portugal, no Departamento de Educação e Ensino a Distância, de que é diretor. Investigador no LE@D (Laboratório de Educação a Distância e eLearning) e no Centro de Filosofia da Universidade de Lisboa. É também diretor no International Board of Standards for Training, Performance and Instruction. Tem uma vasta experiência em ensino e investigação, particularmente no domínio da educação aberta e online, tendo participado em mais de uma vintena de projetos internacionais.

E-mail: Antonio.Teixeira@uab.pt

Branca Miranda. Professora na Universidade Aberta, Portugal, tem uma larga experiência como designer e coordenadora de cursos na modalidade de eLearning na área da Educação. Investigadora do LE@D (Laboratório de Educação a Distância e eLearning) tem participado em projetos de investigação nacionais e internacionais, financiados, relacionados com os MOOCs e da Mobilidade Virtual, sendo autora de publicações em domínios como a utilização de tecnologias na sala aula, a interação em contextos online e a mobilidade virtual.

E-mail: Branca.Miranda@uab.pt

Isolina Oliveira. Professora na Universidade Aberta, Portugal, no Departamento de Educação e Ensino a Distância. Tem larga experiência no desenho e lecionação de cursos graduados e pós-graduados em ambientes virtuais 
de aprendizagem, assim como na investigação em áreas relacionadas com práticas profissionais de professores, avaliação digital e formas de colaboração em contextos online. Desenvolve investigação no Laboratório de Educação a Distância e Elearning (LE@D) da Universidade Aberta.

E-mail: Isolina.Oliveira@uab.pt

Maria do Carmo Pinto.Professora na Universidade Aberta, Portugal, no Departamento de Ciências Sociais e Gestão. Tem vasta experiência em ensino e investigação em educação a distância e eLearning, tendo participado em vários projetos internacionais nestes domínios. Desenvolve investigação no LE@D (Laboratório de Educacão a Distância e eLearning).

E-mail: Maria.Pinto@uab.pt

\section{DIREÇÃO DOS AUTORES}

Universidade Aberta

Rua da Escola Politécnica 141-147 1269-001

Lisboa (Portugal)

Fecha de recepción del artículo: 26/09/2017

Fecha de aceptación del artículo: 21/11/2017

\section{Como citar este artigo:}

Teixeira, A., Miranda, B., Oliveira, I., y Pinto, M. C. (2018). MOOC "Competências digitais para professores": uma prática formativa inovadora. RIED. Revista Iberoamericana de Educación a Distancia, 21(2), pp. 243-261. doi: http://dx.doi. org/10.5944/ried.21.2.19784 